(c) 2010 IEEE. Personal use of this material is permitted. Permission from IEEE must be obtained for all other uses, in any current or future media, including reprinting/republishing this material for advertising or promotional purposes, creating new collective works, for resale or redistribution to servers or lists, or reuse of any copyrighted component of this work in other works. 


\title{
Experimental Demonstration of Focal Plane Array Beamforming in a Prototype Radiotelescope
}

\author{
Douglas Brian Hayman, Member, IEEE, Trevor S. Bird, Fellow, IEEE, Karu P. Esselle, Senior Member, IEEE,
} and Peter J. Hall

\begin{abstract}
Focal plane arrays are being developed to provide dishes with a wide field of view for both the next generation of radiotelescopes and to retrofit existing large radiotelescopes. We describe a prototype radiotelescope, comprising a two dish interferometer with real-time digital beamformer that was built to study focal plane array systems. Two beamformer weightings were applied to the system: A normalized conjugate match and the maximum sensitivity (G/T). Both incorporate the uncorrelated noise from the receiver chains and the latter includes correlated noise from spillover and coupling in the array. A black box approach is taken where the assembled system is considered and the only accessible data is that typically available from an operational radiotelescope. This approach is particularly suitable for complex active antennas where there is insufficient knowledge of the system for beamformer weights to be set a priori. It also allows adaptation to changes such as electronic gain drift, partial failures and alterations in the environment.
\end{abstract}

Index Terms-Active arrays, antenna array feeds, antenna array mutual coupling, antenna measurements, radio astronomy.

\section{INTRODUCTION}

$\mathbf{T}$ HE primary benefit of focal plane arrays (FPAs) to radio astronomy is the increased field of view and hence survey speed. Interest has been developing in arrays with closely spaced elements, which we will refer to as dense FPAs, where the signals from the elements are combined to form beams in contrast to single feed per beam or discretely processed FPAs [1], [2]. These dense FPAs, also known as phased array feeds, can provide superior survey speed and have recently become more attractive due to technological advances.

Discretely processed multibeam FPAs, where the output of each array element forms a separate beam, have been used in

Manuscript received March 04, 2009; revised February 15, 2010; accepted February 16, 2010. Date of publication March 29, 2010; date of current version June 03, 2010.

D. B. Hayman is with Australia Telescope National Facility (ATNF) and Information and Communication Technologies Centre (ICT Centre), Australia's Commonwealth Scientific and Industrial Research Organisation (CSIRO), Epping NSW 1710, Australia and also with Macquarie University, NSW 2109, Australia (e-mail: Douglas.Hayman@csiro.au).

T. S. Bird is with the Information and Communication Technologies Centre (ICT Centre), Commonwealth Scientific and Industrial Research Organisation (CSIRO), Epping NSW 1710, Australia.

K. P. Esselle is with Macquarie University, NSW 2109, Australia.

P. J. Hall is with Curtin University of Technology, Western Australia 6845, Australia.

Color versions of one or more of the figures in this paper are available online at http://ieeexplore.ieee.org.

Digital Object Identifier 10.1109/TAP.2010.2046843 radioastronomy for some time [3], [4]. These systems multiply the field of view available by the number of array elements but they do not provide a contiguous field of view as outlined by Johansson [5]. Veidt shows they typically require four or more interleaved pointings to fully sample the sky [6]. Conversely a dense FPA, where the elements are combined as a complex weighted sum, can fully sample a region of sky, providing contiguous coverage [6]. These feeds also have potentially much wider bandwidths and allow some correction of aberrations present in off-axis beams [6]-[8].

Dense FPAs have come within reach due to progress in a number of areas. Advances in analogue electronics allow the use of uncooled low-noise amplifiers (LNAs) and hundreds of receiver chains per antenna. Improvements in electromagnetic analysis capabilities allow the joint optimization of the array structure and the connecting electronics, especially the LNA and matching components. Finally, developments in digital electronics and devices have made digital beamforming practical, bringing precision, stability and flexibility.

Early work on radioastronomy systems fitted with dense FPAs was done by the National Radio Astronomy Observatory, USA [1], with continuing collaboration with Brigham Young University [9], [10]. ASTRON made early contributions on several fronts including the array design in collaboration with others [11], [12] and beamforming [2]. They continue an active program with an FPA on one of the Westerbork radiotelescope dishes [13]-[15]. The National Research Council of Canada has also pursued this technology [16], reporting recent results of their 180 element prototype [17]. FPAs form an important part of plans for the Square Kilometre Array (SKA) [18]. CSIRO started investigating this area in the early 2000s, and is building the Australian SKA Pathfinder (ASKAP), a radiotelescope with 36 12-m dishes fitted with FPAs [19].

A prototyping test bed for ASKAP was used for our experimental work. This test bed was built to gain experience in receiver, beamformer and system design.

The purpose here is to present results from the prototype radiotelescope, giving a more comprehensive account of beamforming than the early results in conference presentations [20]-[23].

The beamformer weightings used are based on formulations first applied to aperture arrays: The weighting of aperture arrays for maximum gain and G/T is detailed in [24] and [25]. The conjugate field match, with a history in horn feed design [26], has been used as the basis for FPA beamforming [7], [27], [28]. This approach assumes the array elements are point field samplers and does not usually include mutual coupling effects. By 
contrast the maximum gain or maximum directivity weightings do include these effects and have been applied to FPAs by Bird [29] and Lam et al. [30]. The maximum G/T has been applied to FPAs by Bird and Hayman [31], Brisken [32] and Hansen et al. [33] and Jeffs et al. [34] among others.

The design of FPAs requires a full understanding of the system, including a detailed model of the electromagnetics of the FPA and its relationship to the reflector and the receiving system [32], [35], [36]. During development of an array, it is common to measure the scattering matrix and radiation patterns of the array separately from the receiver electronics. With knowledge of the characteristics of the electronics, the overall performance of the array can be predicted.

However once the system is assembled, access to internal ports is usually impractical, particularly once the array is integrated with the reflector. Therefore, a black-box approach was adopted for this work. We consider the situation where there the system can operate in two modes. Firstly where there is access to the beamformed output and secondly where each element's signal can be correlated against the others.

The implementation used is presented here to make it clear how the weights are calculated and how they relate to common figures of merit.

The next section discusses the implementation of the weighting formulation. Section III describes the prototype radiotelescope. The measurement details are provided in Section IV and they are discussed in Section V. Finally in Section VI, conclusions are drawn about the two beamformer weightings and the evaluation techniques.

\section{IMPLEMENTATION}

The standard definition of antenna gain [37] includes the dissipative losses in the antenna and does not include the match between the receiver and the antenna. When measuring an assembled active antenna such as a focal plane array, however, it is impossible to separate these effects from the electronic gain in the receiver electronics. The noise contributions of the antenna and receiver also cannot easily be separated. In the black box approach adopted (Fig. 1), it is convenient to define the reference plane for the gain and system noise as the radiation port, consequently directivity is used as the reference for $\mathrm{G} / \mathrm{T}$ evaluation. Dissipative losses in the antenna are assigned to the receiver. Some other approaches that assume modelled or measured knowledge of parameters internal to the active antenna are given in [38]-[41].

The voltage at the beamformer output, with the assumption that the array system is linear, is given by

$$
v_{\text {out }}(t)=\sum_{i=1}^{n}\left(\frac{\lambda}{\sqrt{2 \eta_{0}}} E_{p, i}(t) e_{p, i}+v_{T_{\mathrm{sys}}, i}(t)\right) w_{i}
$$

where $\lambda$ is the wavelength; $\eta_{0}$ the impedance of free space; $E_{p, i}(t)$ the field strength of the desired signal in the co-polarization, denoted by subscripts $p$ and with unit vector definition $\hat{p}$; $e_{p, i}(\theta, \phi)=\vec{e}_{p, i}(\theta, \phi) \cdot \hat{p}$, the co-polarized embedded-element field-pattern (with final system loading, not open or shorted); $v_{T_{\mathrm{sys}}, i}$, the noise from all sources referred to the input of each el-

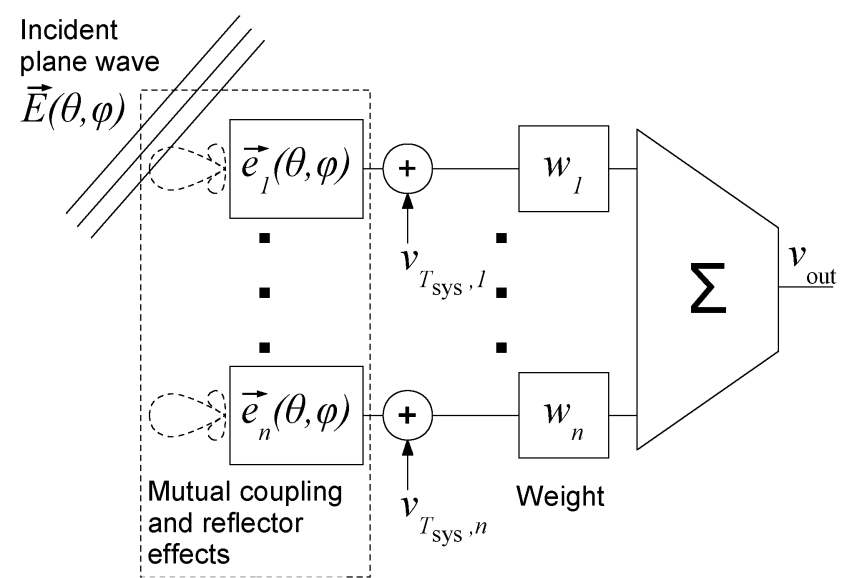

Fig. 1. FPA signal and noise model showing $n$ beamformed focal plane array elements. The parameters are: $\vec{E}$ : desired incident plane-wave. $\vec{e}_{i}$ : embeddedelement field-pattern. $v_{T_{\mathrm{sys}}, i}:$ noise from all sources referred to the input of each element. $w_{i}$ : adjustable complex weight.

ement; and $w_{i}$, the adjustable complex weight. ${ }^{1}$ The subscript $q$ and unit vector $\hat{q}$ correspond to the cross-polarized components and $e_{q, i}(\theta, \phi)$ is the cross-polarized embedded field-pattern. The constants have been chosen so that $\left|v_{T_{\mathrm{sys}}, i}\right|^{2}$ is the noise power and the directivity of the $i^{\text {th }}$ element (i.e. $w_{i}=1$ and $w_{j}=0$, $\forall j \neq i$ ) is

$$
D_{p, i}=4 \pi e_{p, i}^{*} e_{p, i}
$$

This formulation leads the $e_{p, i}$ and $e_{q, i}$ to include pattern distortion due to mutual coupling but not coupling loss or dissipative loss.

By using superposition it can be readily shown that the directivity of the array for the $\hat{p}$ polarization is

$$
D_{p}=4 \pi \frac{\mathbf{w}^{H} \mathbf{e}_{p}^{*} \mathbf{e}_{p}^{T} \mathbf{w}}{\mathbf{w}^{H} \mathbf{C}_{e} \mathbf{w}}
$$

where

$$
\left[\mathbf{C}_{e}\right]_{i j}=\iint_{\Omega}\left(e_{p, i}^{*} e_{p, j}+e_{q, i}^{*} e_{q, j}\right) d \Omega
$$

and $\mathbf{e}_{p}=\left[\cdots e_{p, i} \cdots\right]^{T}$ and $\mathbf{w}=\left[\cdots w_{i} \cdots\right]^{T}$ are column vectors representing the array field-patterns and weights respectively. The superscript $\mathrm{T}$ denotes the transpose and $\mathrm{H}$ the Hermitian or conjugate transpose.

The equivalent system temperature can be expressed as

$$
T_{\mathrm{sys}}=\frac{\mathbf{w}^{H} \mathbf{C}_{T_{\mathrm{sys}}} \mathbf{w}}{\mathbf{w}^{H} \mathbf{C}_{e} \mathbf{w}}
$$

where $\mathbf{C}_{T_{\mathrm{sys}}}$ is the noise covariance matrix for the array. That is the elements of $\mathbf{C}_{T_{\text {sys }}}$ are

$$
\left[\mathbf{C}_{T_{\mathrm{sys}}}\right]_{i j}=\mathrm{E}\left\{v_{T_{\mathrm{sys}}, i}^{*}(t) v_{T_{\mathrm{sys}}, j}(t)\right\}
$$

\footnotetext{
${ }^{1}$ Another common notational convention uses the conjugate of the beamformer weights.
} 
where $\mathrm{E}\{\}$ is the expected value and can be measured by correlating element pairs.

The system temperature is the sum of noise from the antennareceiver combination and radiation from the surrounding scene: $\mathbf{C}_{T_{\mathrm{sys}}}=\mathbf{C}_{T_{\mathrm{rx}}}+\mathbf{C}_{T_{\mathrm{scene}}}$. Modelling the contributors to $\mathbf{C}_{T_{\mathrm{sys}}}$ is described in other FPA treatments such as [34], [35], [42], [43].

Using (3) and (5) the G/T for the system is then

$$
G_{p} / T=\frac{D_{p}}{T_{\mathrm{sys}}}=4 \pi \frac{\mathbf{w}^{H} \mathbf{e}_{p}^{*} \mathbf{e}_{p}^{T} \mathbf{w}}{\mathbf{w}^{H} \mathbf{C}_{T_{\mathrm{sys}}} \mathbf{w}} .
$$

The weights for the maximum G/T are [25], [44]

$$
\mathbf{w}=\mathbf{C}_{T_{\mathrm{sys}}}^{-1} \mathbf{e}_{p}^{*} \text {. }
$$

\section{A. Measurable Quantities}

The model outlined above established the formulation for the maximum $\mathrm{G} / \mathrm{T}$ weighting, however, in an assembled system there is usually insufficient information to determine accurate values of the embedded element patterns $e_{p, i}$. We will show how the maximum $\mathrm{G} / \mathrm{T}$ weighting can be found from data that is readily measured - the covariance matrix and the element responses to a distant point source-as used, e.g., in [34]. We will also detail a simpler weighting that does not require the covariance matrix, referred to here as the normalized conjugate match.

To establish the implementation based on measurable quantities, the unknown contributions of electronic gain, scattering by the antenna, dissipative losses in the antenna, mutual coupling losses and mismatch effects are assigned to a set of complex terms $h_{i}$. A new weight vector $\mathbf{u}: u_{i}=w_{i} / h_{i}$ is used to represents the weights actually applied in the beamformer.

Using (1) the voltage at the beamformer output is given by

$$
v_{\text {out }}(t)=\sum_{i=1}^{n}\left(\frac{\lambda}{\sqrt{2 \eta_{0}}} E_{p, i}(t) \dot{e}_{p, i}+\hat{v}_{T_{\mathrm{sys}}, i}(t)\right) u_{i}
$$

where $\hat{e}_{p, i}=h_{i} e_{p, i}$ is the active element response to a distant point source and $\hat{v}_{T_{\mathrm{sys}}, i}=h_{i} v_{T_{\mathrm{sys}}, i}$ is the noise voltage at the beamformer output for unity weighting for the $i^{\text {th }}$ element (i.e. $u_{i}=1$ and $u_{j}=0, \forall j \neq i$ ).

This modified model will be used to show that the maximum $\mathrm{G} / \mathrm{T}$ weighting equivalent to (8) can be determined even though the electronic gains and the losses listed above are not directly known.

The relative magnitudes and phases of the $e_{i}$ are determined from the correlation product from the interferometer pointed at a point source. The noise covariance matrix, scaled for the weight vector $\mathbf{u}$, is

$$
\left[\mathbf{C}_{T_{\mathrm{sys}}, u}\right]_{i j}=\mathrm{E}\left\{\hat{v}_{T_{\mathrm{sys}}, i}^{*}(t) \hat{v}_{T_{\mathrm{sys}}, j}(t)\right\}=h_{i}^{*} h_{j}\left[\mathbf{C}_{T_{\mathrm{sys}}}\right]_{i j} .
$$

Using the relationships between $\mathbf{w}$ and $\mathbf{u}$ and between $\mathbf{e}$ and é, (7) can be expressed in terms of $\mathbf{u}$ and é, giving

$$
G_{p} / T=\frac{D_{p}}{T_{\mathrm{sys}}}=4 \pi \frac{\mathbf{u}^{H} \dot{\mathbf{e}}_{p}^{*} \dot{\mathbf{e}}_{p}^{T} \mathbf{u}}{\mathbf{u}^{H} \mathbf{C}_{T_{\mathrm{sys}}, u} \mathbf{u}}
$$

As with (8), the weights for the maximum G/T are

$$
\mathbf{u}=\mathbf{C}_{T_{\mathrm{sys}}, u}^{-1} \mathbf{e}_{p}^{*} .
$$

This expression allows the maximum G/T weighting to be found from the conveniently measured data $e_{i}$ and $\hat{v}_{T_{\mathrm{sys}}, i}$.

Initially the full covariance matrix was not available from the system and so a simpler weighting was employed. The conjugate match to the desired signal was normalized by the noise from each element, that is the diagonal of the noise covariance matrix and so the weighting terms are

$$
\mathbf{u}=\left[\cdots \hat{e}_{p, i}^{*} /\left[\mathbf{C}_{T_{\mathrm{sys}}, u}\right]_{i i} \cdots\right]^{T} .
$$

The equivalent weights in terms of the field patterns and noise covariance matrix referred to the radiation port are

$$
\mathbf{w}=\left[\cdots e_{p, i}^{*} /\left[\mathbf{C}_{T_{\mathrm{sys}}}\right]_{i i} \cdots\right]^{T} .
$$

The similarity between this normalized conjugate match and the maximum gain is described in Appendix B. The normalized conjugate match and the maximum G/T weightings are equivalent if the noise from each element is uncorrelated.

With this black box approach, it was possible to obtain the data for calculating the weights using the completed radiotelescope system. The calculation of the maximum G/T weighting (12) requires the element responses to a distant point source $e_{p, i}$ and the noise covariance matrix $\mathbf{C}_{T_{\mathrm{sys}}, u}$ with the antenna pointed away from any strong sources (off-source).

\section{INSTRUMENTATION}

The performance of a prototype radio telescope with various beamformer weightings has been studied. The radiotelescope comprised a two dish interferometer established at the CSIRO Radiophysics Laboratory in Sydney. It had a $90 \mathrm{~m}$ baseline, oriented approximately east-west (Figs. 2 and 3). The two dishes have a diameter of $14.2 \mathrm{~m}$ and an F/D of 0.40. The western dish was fitted with a single horn with an edge taper of $17 \mathrm{~dB}$. The eastern dish was fitted with a single polarization $8 \times 8$ array of Vivaldi elements, originally used in the ASTRON THEA project [45]. The signals were transported from the FPA on coaxial cables to receivers in the pedestal. The $70 \mathrm{MHz}$ intermediate frequency signals from the receiver ( $24 \mathrm{MHz}$ wide) were then fed into the 24 input digital beamformer-correlator.

Twenty one of the sixty four elements of the array were selected for beamforming. Fig. 4 shows the positions from above the dish and feed, the dominant polarization is electric field horizontal, west is in the top left corner and north the top right. The initial more central element selection was used first and is shown in Fig. 4(b). Element number seven developed a fault and so the connections were then moved to an offset selection for the latter measurements (Fig. 4(e)). This moved the beam $1.8^{\circ}$ away from the reflector boresight. With this set of connections, element 17 developed an intermittent fault. Being at the edge of the selected area it was expected to have less impact than element seven in 


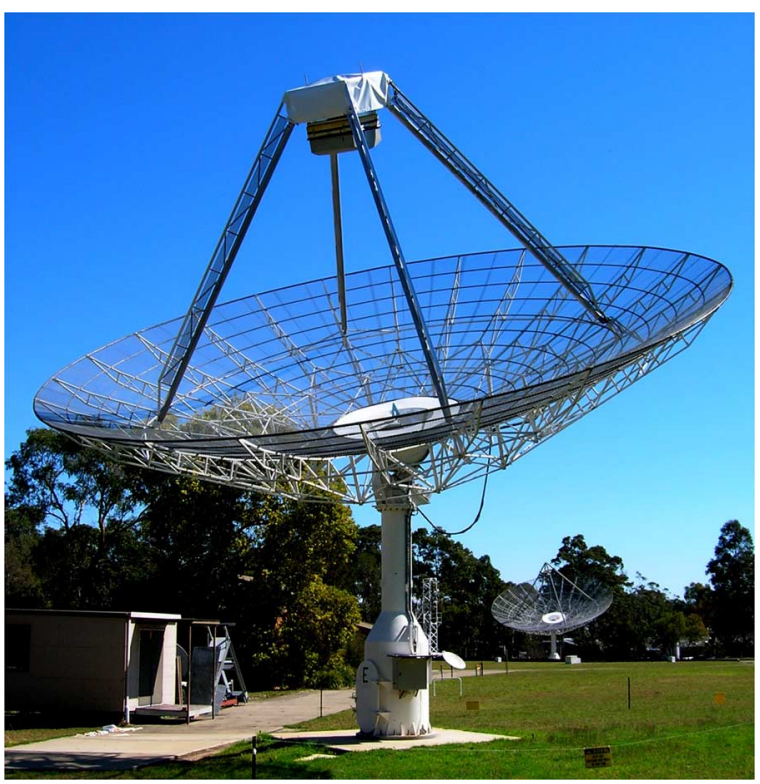

Fig. 2. The two dish prototype radiotelescope located in Sydney; the east antenna in the foreground is fitted with the FPA and the west antenna in the background with a single horn.

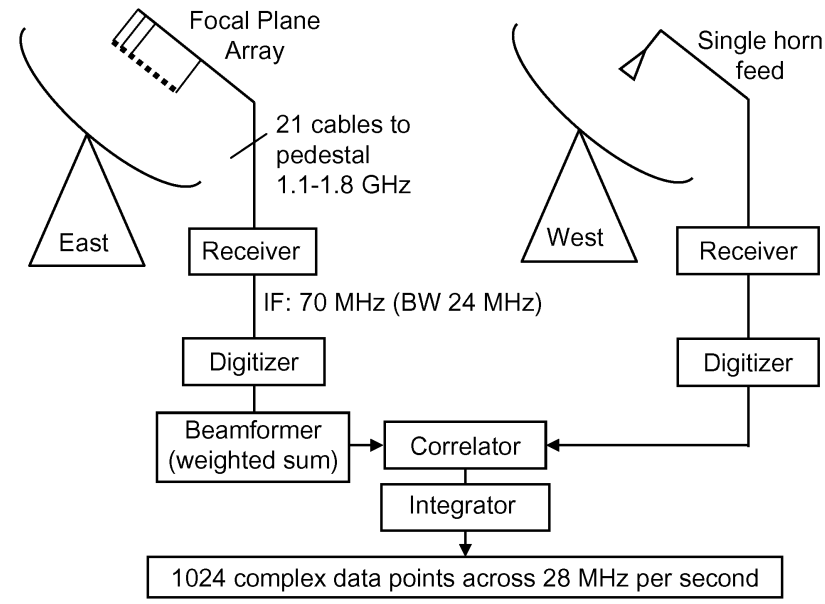

Fig. 3. System diagram of the prototype radiotelescope. Twenty one signals are brought down from the FPA through the receiver to the digitizer. Autocorrelations and cross-correlations between any two FPA inputs were also possible.

the central element selection. The other three beamformer inputs were used for the reference (western) antenna feed and the two vertex noise sources used for calibration.

The original FPA range of 700 to $1800 \mathrm{MHz}$ was found to be too wide with significant distortion products present from the severe radio frequency interference (RFI) environment of suburban Sydney. An 1150 to $1750 \mathrm{MHz}$ filter was fitted to each element between the first and second amplification stages, leaving a few RFI free bands available for measurements. The center frequency of $1200 \mathrm{MHz}$ was chosen for the results presented in this paper. At this frequency the element spacing was 0.51 wavelengths and with a higher frequency, with element lower spacing, a single element would dominate and beamforming would have less effect.

The separate receiver chains result in drift of the gain and phase for each element relative to the others. To correct for this a noise signal was radiated from the vertex of the dish by a noise source connected to a small antenna. With the noise source turned on and a coupled signal from the source fed into the correlator, a reference phase was established and this was used to compensate for drift. The noise source used in this instance did not have a stabilized output level and so the off-source autocorrelation of each element was used as a measure of the magnitude drift instead. A stabilized noise source is recommended for future systems.

Where an interferometer, with its higher sensitivity, is unavailable, a single dish can be used to obtain the beamformer coefficients and determine the G/T performance [17], [34], [46]. [22].

The prototype radiotelescope is described in more detail in

\section{MEASUREMENTS}

A series of investigations were conducted on the prototype radiotelescope to demonstrate beamforming and evaluation methods. For each set of measurements, there were two phases: First the data required for calculating the array weights was gathered and then tests were undertaken to evaluate the system with beamforming applied.

\section{A. Beamformer Weight Data Collection}

The noise covariance matrix (Section II) was used for determining the system noise for different sets of weights. To generate the matrix, the antenna was pointed off-source, i.e. away from any strong sources whilst retaining a similar surrounding scene of radiative noise. This was achieved by keeping the elevation approximately the same for the on-source and off-source measurements.

The diagonal terms of this matrix dominated, being at least ten times the off diagonal terms. The correlation coefficients are used for this comparison $r_{i j}=c_{i j} / \sqrt{c_{i i} c_{j j}}$ where $c_{i j}=$ $\left[\mathbf{C}_{T_{\mathrm{sys}}, u}\right]_{i j}$. Element pairs that were adjacent in the H-plane (e.g., elements 4 and 8, Fig. 4) had correlation terms between 6 and $10 \%$ of the diagonals. Element pairs that were adjacent in the E-plane had much lower terms. This corresponds to the degree of coupling that is expected with electric-source dominated elements [47].

Initially the system was not equipped to measure $\mathbf{C}_{T_{\mathrm{sys}}, u}$ and only the normalized conjugate match weighting was used for the central element selection.

In interferometer mode, both dishes were pointed at a suitable radio source and the correlations of each element against the reference (western) antenna were recorded. The galaxy M87 (Virgo A) was chosen for this purpose because it appeared as a point source for the $90 \mathrm{~m}$ baseline at $1200 \mathrm{MHz}$, it has a high flux density and it is available for $71 / 2 \mathrm{hrs}$ a day for the location and range of motion of these antennas.

\section{B. Evaluation of the Beamformed System}

The weights were calculated from the noise covariance matrix and the point source response data (see (12) and (13)). The single element in the center of the 21 selected elements was used as a reference for the weighting cases (number one in Fig. 4. It is stressed that this single element over illuminates the reflector and so a purpose designed horn, such as the one on the 


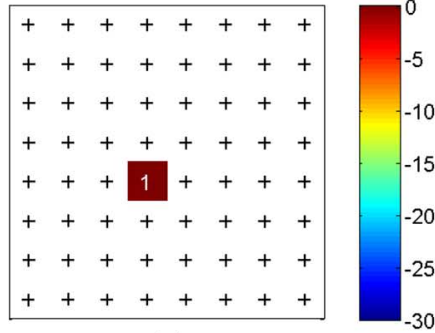

(a)

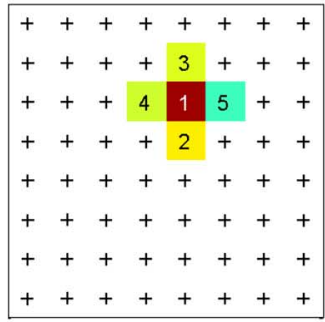

(d)

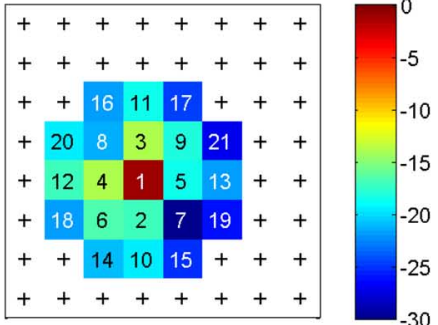

(b)

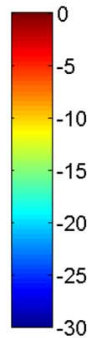

(e)

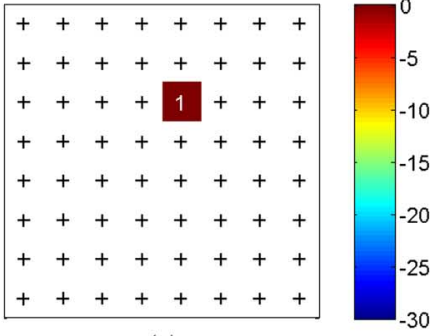

(c)

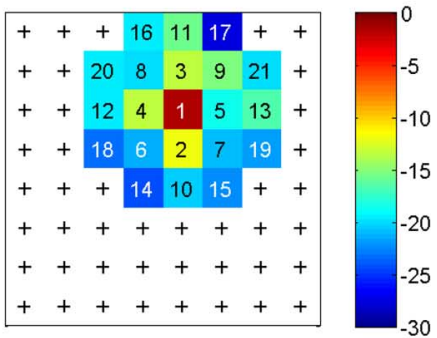

(f)

Fig. 4. Magnitude of array element weights (decibels) at $1200 \mathrm{MHz}$ for the weighting cases applied, showing the central and offset element selections. The E field polarization of the array is horizontal. (a) Single element—central. (b) Normalized conjugate match, 21 elements—central. (c) Single element—offset. (d) Maximum G/T, 5 elements—offset. (e) Normalized conjugate match, 21 elements—offset. (f) Maximum G/T, 21 elements—offset.

western antenna, would perform better in both noise and efficiency. Nonetheless, the single element was convenient to use as it was expected to have a similar noise contribution from the receiver electronics as the weighted array and its G/T would not change too much over time. Tests were also done with five elements to see the effect of the reduction from 21 elements.

The weighting cases applied were the normalized conjugate match with 21 elements for both the central and offset element selections and the maximum G/T with five and 21 elements in the offset selection. Fig. 4 shows the magnitude of the calculated weights for the cases presented here.

Radiation patterns were obtained with the system acting as an interferometer using a point source. The correlator output is the product of the voltage gains of the eastern and western dishes. The reference (western) dish tracks the source and the eastern dish changes its pointing relative to the source to make the pattern cuts. M87 was used for the central element selection. Sagittarius $A^{*}$, being higher in the sky allowed a greater range in declination, was used for the offset element selection. Cuts were made in the $\mathrm{E}, \mathrm{H}, 45^{\circ}$ and $135^{\circ}$ planes at $0.25^{\circ}$ spacing over $8^{\circ}$ and $10^{\circ}$ spans for the central and offset selections respectively (Fig. 5). The $45^{\circ}$ plane (with respect to the E-field) corresponds to cuts in declination (north-south) and the $135^{\circ}$ plane corresponds to cuts in hour angle (east-west). These patterns were interpolated using the formulation described in Bucci [48].

The half-power beamwidth (HPBW) is about $1.7^{\circ}$ with the variations shown in Table I.

A readily accessible method was used to compare single element and beamformed $\mathrm{G} / \mathrm{T}$ performance. A directivity measure was obtained from the interferometer response to M87 and a noise measure was obtained from the autocorrelation of weighted signal off-source. These measures do not represent the true directivity or system temperature as they do not include the overlap integral terms in the denominators of (3) and (5).
Their ratio however is proportional to the true G/T as shown by (7) and (11).

Table I shows the G/T improvement seen for the beamformed cases over the single element cases. These measurements were repeated a number of times and the results vary by about $0.1 \mathrm{~dB}$ but the difference between the normalized conjugate match and the maximum $\mathrm{G} / \mathrm{T}$ case remains within $0.03 \mathrm{~dB}$ of the figures quoted.

The absolute G/T values for the offset element selection were determined by measuring the ratio of the power pointing at and away from a source of known strength. The G/T value then is found by

$$
G / T=4 \pi \frac{2 k_{\mathrm{B}}\left(Y_{\mathrm{src}}-1\right)}{\lambda^{2} \mathbb{S}}
$$

where $k_{\mathrm{B}}$ is the Boltzmann constant, $Y_{\mathrm{src}}$ is the on-source/offsource power ratio and $\mathbb{S}$ is the spectral power flux density of the source.

M87 was used as the source and its strength was determined by extrapolating the data from Ott et al. [49], giving the value of $\sim 230 \pm 16 \mathrm{Jy}$ at $1200 \mathrm{MHz}$. The uncertainty in $Y_{\mathrm{src}}, 0.02$ $\mathrm{dB}$, dominates the combined uncertainty of $\sim 0.7 \mathrm{~dB}(\sim 17 \%)$. The combined uncertainties quoted in this paper are calculated from the root sum of squares of the contributing uncertainties and represent the $\sim 95 \%$ probability interval. The system temperature over aperture efficiency (based on the dish area), $\eta_{\mathrm{ap}}$, is also presented as the figure of merit as this is a useful way of comparing feed systems:

$$
\frac{T_{\text {sys }}}{\eta_{\text {ap }}}=\frac{A S}{2 k_{\mathrm{B}}\left(Y_{\text {src }}-1\right)}
$$

where $A$ is the dish area. Both figures of merit are shown in Table I. 


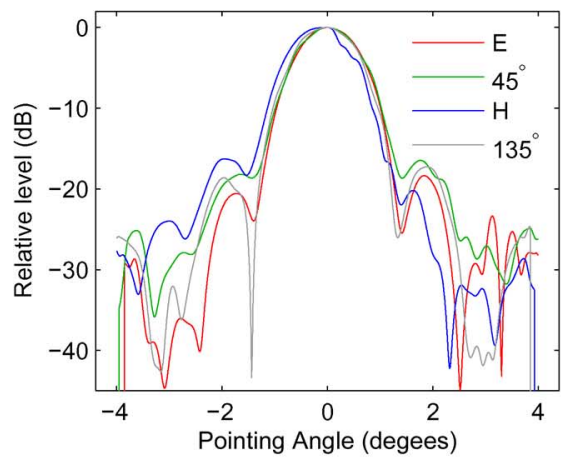

(a)

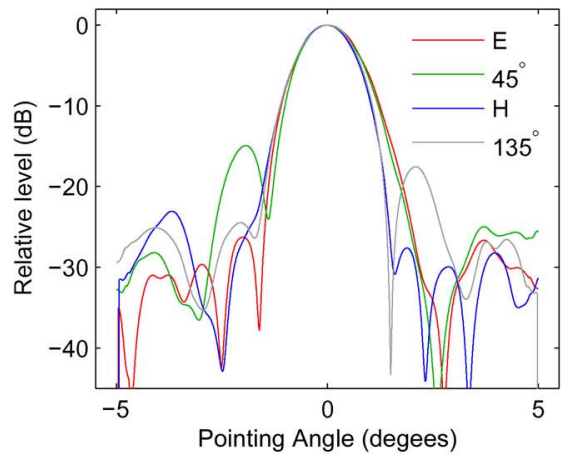

(d)

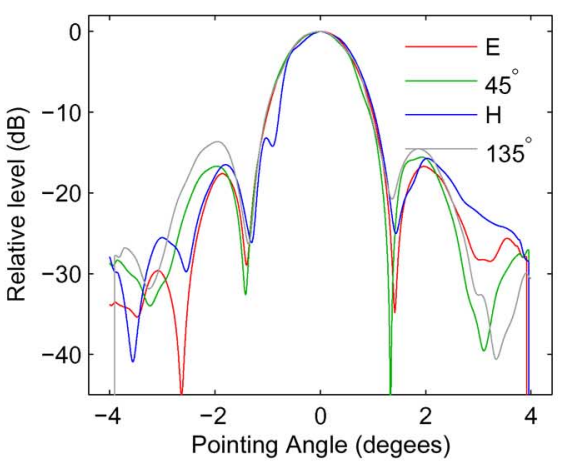

(b)

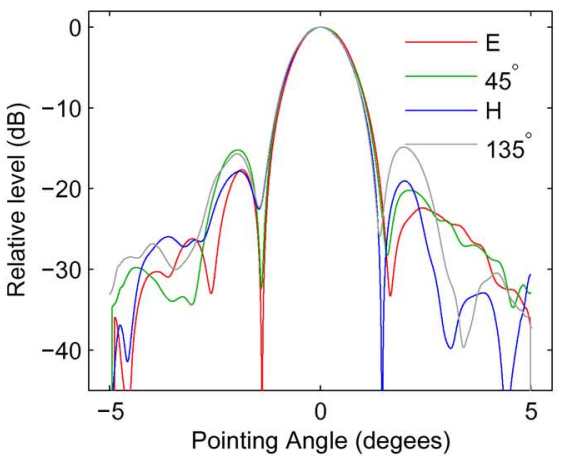

(e)

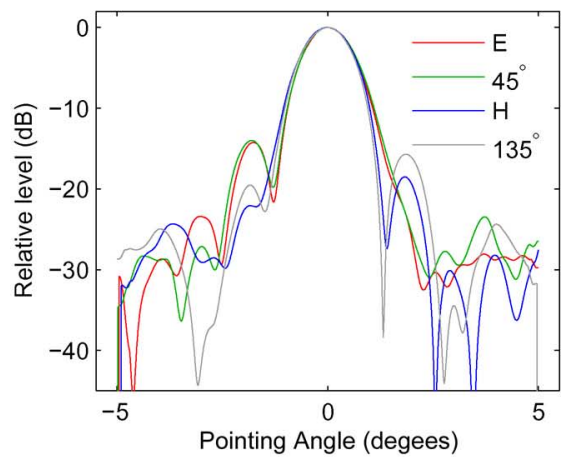

(c)

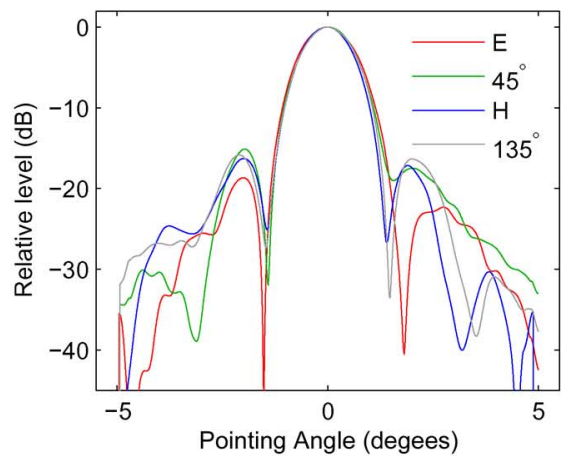

(f)

Fig. 5. Radiation pattern cuts at $1200 \mathrm{MHz}$ for the weighting cases applied. (a) Single element—central. (b) Normalized conjugate match, 21 elements—central. (c) Single element—offset. (d) Maximum G/T, 5 elements—offset. (e) Normalized conjugate match, 21 elements—offset. (f) Maximum G/T, 21 elements—offset.

TABLE I

BEAMFORMED PERFORMANCE AT $1200 \mathrm{MHz}$

\begin{tabular}{|c|c|c|c|c|c|c|c|c|c|c|}
\hline \multirow[b]{2}{*}{$\begin{array}{l}\text { Element } \\
\text { sel. }\end{array}$} & \multirow[b]{2}{*}{ Weighting } & \multirow[b]{2}{*}{$\begin{array}{l}\text { Num. } \\
\text { elts. }\end{array}$} & \multirow[b]{2}{*}{$\begin{array}{c}\Delta \mathrm{G} / \mathrm{T}^{a} \\
(\mathrm{~dB})\end{array}$} & \multirow[b]{2}{*}{$\begin{array}{c}\text { HPBW } \\
\left(^{\circ}\right)\end{array}$} & \multicolumn{3}{|c|}{ On/Off M87 } & \multicolumn{2}{|c|}{ Absorber } & \multirow[b]{2}{*}{$\begin{array}{l}\eta_{\mathrm{ap}} \\
(\%)\end{array}$} \\
\hline & & & & & $\begin{array}{l}Y_{\text {src }} \\
(\mathrm{dB})\end{array}$ & $\begin{array}{c}G / T \\
(\mathrm{~dB}(1 / \mathrm{K}))\end{array}$ & $\begin{array}{c}T_{\text {sys }} / \eta_{\mathrm{ap}} \\
(\mathrm{K})\end{array}$ & $\begin{array}{l}Y_{\text {abs }} \\
(\mathrm{dB})\end{array}$ & $\begin{array}{c}T_{\text {scene }} \\
(\mathrm{K})\end{array}$ & \\
\hline central & single & 1 & 0 & 1.16 & & & & & & \\
\hline central & norm. conj. & 21 & 1.98 & 1.18 & & & & & & \\
\hline offset & single & 1 & 0 & 1.17 & 0.09 & 17.2 & 614 & 2.43 & 112 & 41 \\
\hline offset & $\max . \mathrm{G} / \mathrm{T}$ & 5 & 1.15 & 1.27 & 0.13 & 18.8 & 419 & 3.31 & 66 & 49 \\
\hline offset & norm. conj. & 21 & 2.02 & 1.22 & 0.16 & 19.6 & 350 & 3.36 & 64 & 57 \\
\hline offset & $\max . \mathrm{G} / \mathrm{T}$ & 21 & 2.08 & 1.24 & 0.16 & 19.5 & 357 & 3.57 & 55 & 54 \\
\hline
\end{tabular}

${ }^{a} \Delta \mathrm{G} / \mathrm{T}$ refers to the ratio of the $\mathrm{G} / \mathrm{T}$ with the weighting and the corresponding single element $\mathrm{G} / \mathrm{T}$.

A measure of the system temperature can be arrived at by placing microwave absorber under the feed. A $1.8 \mathrm{~m} \times 1.8 \mathrm{~m}$ sheet of $457 \mathrm{~mm}$ thick absorber was used. The power ratios for with and without absorber, $Y_{\text {abs }}$, are shown in Table I; the uncertainty is $\sim 0.02 \mathrm{~dB}$. The interpretation of these results requires knowing either the receiver system temperature or the noise contribution from the surrounding scene [50]. An estimate of the former is detailed in Section V-B.

\section{Discussion}

The measurements are used to interpret the behavior of the beamforming weights in the prototype radiotelescope. Characteristics of the aperture distribution are determined from the radiation patterns. An estimate of the FPA noise temperature is calculated and then used to find the spillover temperatures and aperture efficiencies from the power ratio measurements.

\section{A. Radiation Patterns}

As a general observation, it is apparent from the pattern distortion that the H-plane radiation pattern cuts for the single and beamformed central element selection (Fig. 5(a) and (b)) suffered minor contamination with noise. This is probably due to interference being present at the moment of the distorted data in those cuts.

The patterns in the $\mathrm{E}$ and $45^{\circ}$ planes show a coma effect in the single and five-element cases for the offset element selection (Fig. 5(c) and (d)). However in the 21-element cases the effect is reduced (Fig. 5(e) and (f)). This improvement demonstrates the ability of FPAs to compensate for such off-axis aberrations [51].

To understand beamforming behaviour, it is valuable to determine the aperture field distribution generated by the feed patterns. Aperture distributions are commonly ascertained using microwave holography from an extensive 2D far-field map. This 


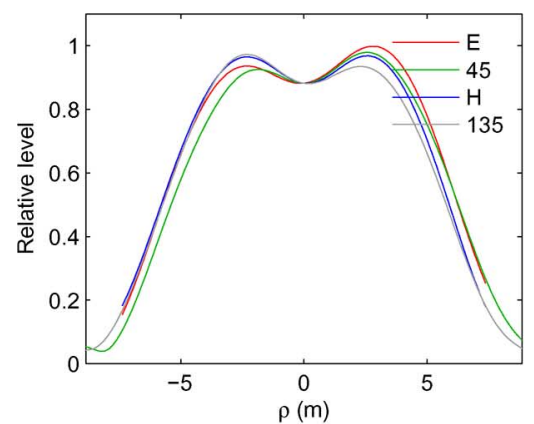

(a)

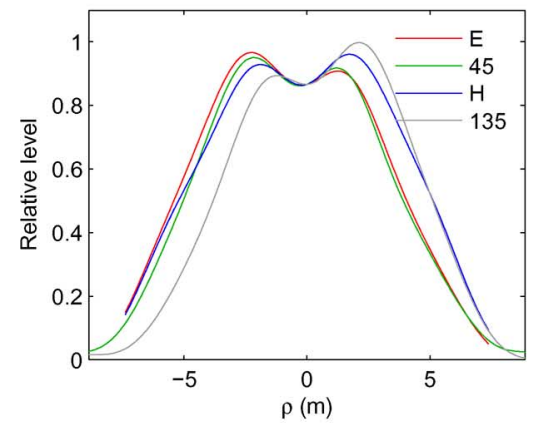

(d)

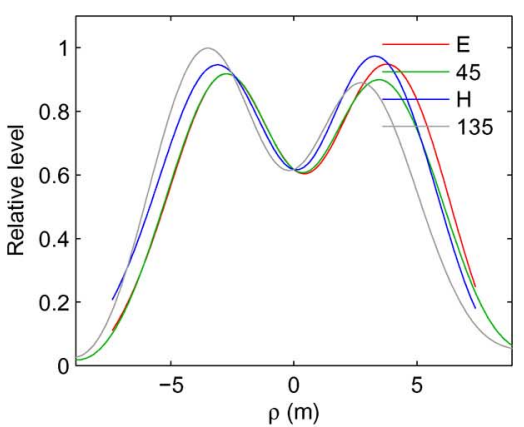

(b)

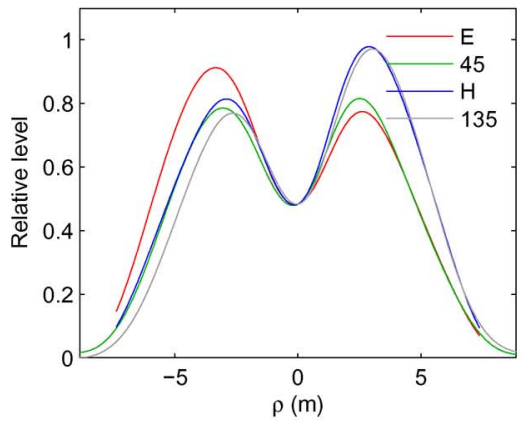

(e)

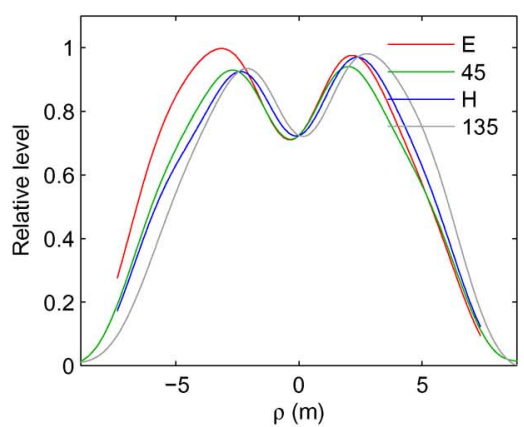

(c)

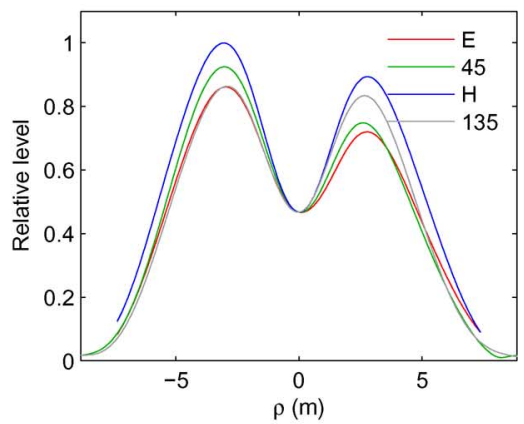

(f)

Fig. 6. Aperture illumination cuts at $1200 \mathrm{MHz}$, magnitude (dB). (a) Single element—central. (b) Normalized conjugate match, 21 elements—central. (c) Single element—offset. (d) Maximum G/T, 5 elements—offset. (e) Normalized conjugate match, 21 elements—offset. (f) Maximum G/T, 21 elements—offset.

approach was not available here and so the technique described in Appendix A is used.

The magnitude and phase distributions from the measured cases are shown in Figs. 6 and 7 respectively. The magnitude plots show a slight narrowing of the feed pattern for the beamformed cases over the single element cases. The phase plots are flatter in the beamformed cases. This is most likely due to the phase center of the array elements being slightly above or below the focal plane (defocused) and the beamforming correcting the location of the phase center.

In the central element selection the first sidelobe levels are higher for the beamformed case (Fig. 5(b)) than for the single element (Fig. 5(a)). The aperture distributions show an increase in aperture taper for the beamformed case (cf. Fig. 6(a) and (b)) and normally this reduces the sidelobe levels. However the concentration of the feed pattern under the feed blockage appears to be the factor increasing the sidelobes [52].

A tradeoff to be considered with the use of FPAs is the increased blockage. On this instrument, the feed, occupying 2.1 $\mathrm{m}^{2}$, blocks $1.3 \%$ of the physical aperture, corresponding to a $\sim 5 \%$ reduction in gain [52]. The struts, occupying $3.4 \mathrm{~m}^{2}$, block $2.1 \%$ of the physical aperture corresponding to a $\sim 4 \%$ reduction in gain.

\section{B. G/T Results}

The equivalent receiver noise temperature $T_{\mathrm{rx}}$ of the FPA was calculated to aid in the understanding of the $\mathrm{G} / \mathrm{T}$ results. This was calculated as $137 \mathrm{~K}$ using estimates of component contributions, listed in Table II, obtained from both the FPA supplier and data from the modifications performed by CSIRO. Note that $T_{\mathrm{rx}}$ includes dissipative losses in the antenna but not radiation from the surrounding scene, such as spillover. Measurements on an array with the same elements and LNAs but with a differing subsequent receiver chain produced a very similar result [53].

Rough uncertainties assigned to the values contributing to this estimate show the two dominant contributors to the total uncertainty in $T_{\mathrm{rx}}$ are the dissipative loss in the antenna and the LNA noise. The former is assigned $95 \%$ confidence interval bounds of -0.1 and $+0.2 \mathrm{~dB}$, based on our experience with similar calculations and measurements, resulting in a -10 to $+20 \mathrm{~K}$ uncertainty in $T_{\mathrm{rx}}$ and the latter bounds of -9 and $+18 \mathrm{~K}$, based on the manufacturers data sheet and resulting in contributions of -9 and $+18 \mathrm{~K}$ to the $T_{\mathrm{rx}}$ uncertainty. The upper and lower bounds are combined as a root sum of squares separately. The combined 95\% confidence interval bounds for $T_{\mathrm{rx}}$ are $-17 \mathrm{~K}$ $(120 \mathrm{~K})$ and $+29 \mathrm{~K}(166 \mathrm{~K})$. The relatively high combined uncertainty in reflects the incomplete nature of the information readily available for this FPA.

The estimated value of the receiver equivalent noise temperature, $T_{\mathrm{rx}}=137 \mathrm{~K}$, was used to give representative values of the equivalent noise temperature from the surrounding scene for the offset element selection. This is given by

$$
T_{\text {scene }}=\frac{T_{\mathrm{abs}}-T_{\mathrm{rx}}\left(Y_{\mathrm{abs}}-1\right)}{Y_{\mathrm{abs}}}
$$

where $T_{\mathrm{abs}}$ is the temperature of the absorber, $299 \mathrm{~K}$, and $Y_{\mathrm{abs}}$ is the power ratio. These results are shown in Table I. The uncertainty in $T_{\text {scene }}$ is dominated by $T_{\mathrm{rx}}$. This varies slightly for the different measurement sets with the worst case being \pm 16 $\mathrm{K}$. The uncertainty is much less for the relative values however: $T_{\text {scene }}$ for the five-element maximum G/T case is $46 \pm 3 \mathrm{~K}$ lower than the single element case. $T_{\text {scene }}$ for the 21-element maximum $\mathrm{G} / \mathrm{T}$ case is $58 \pm 4 \mathrm{~K}$ lower than the single element 


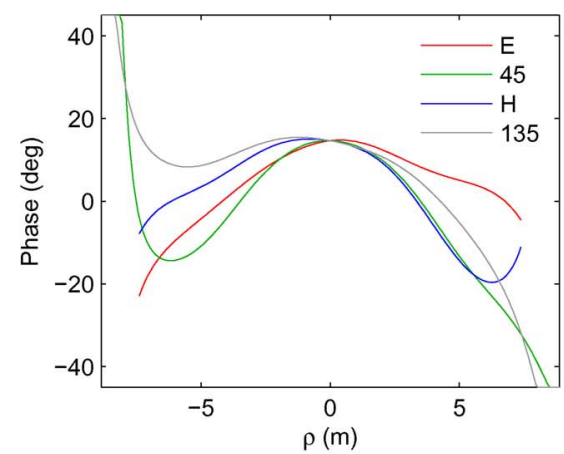

(a)

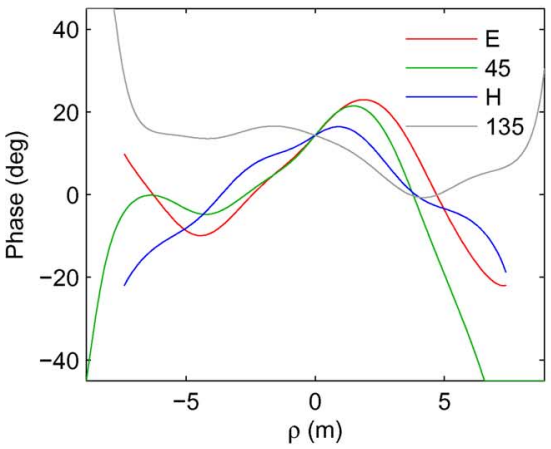

(d)

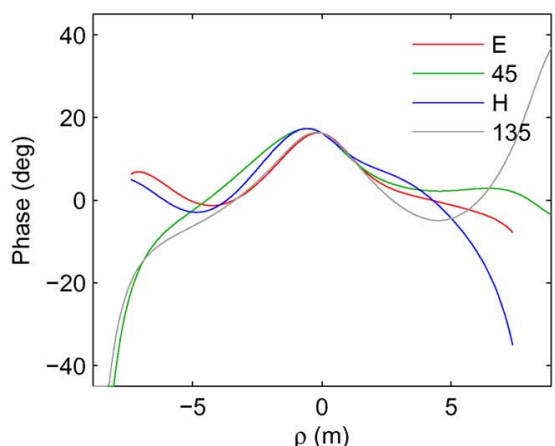

(b)

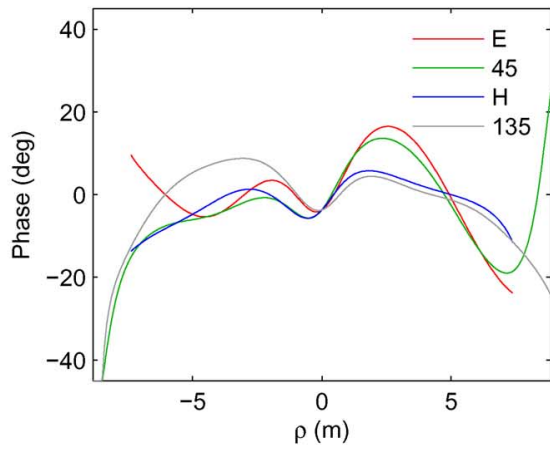

(e)

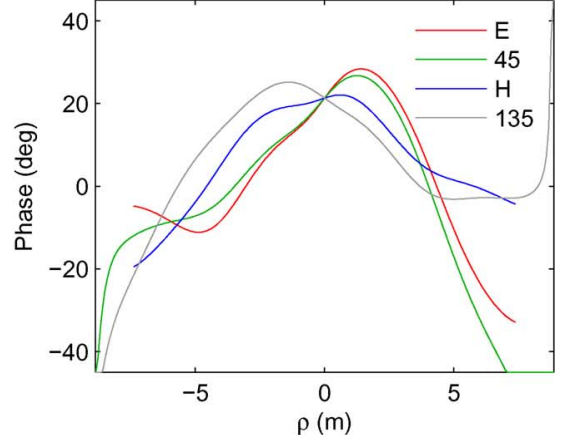

(c)

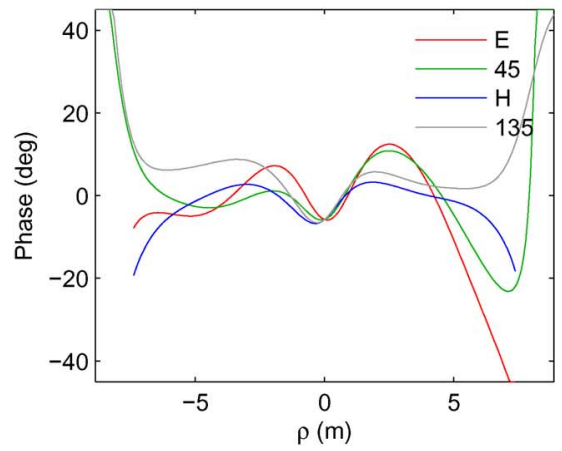

(f)

Fig. 7. Aperture illumination cuts at $1200 \mathrm{MHz}$, phase (deg). (a) Single element—central. (b) Normalized conjugate match, 21 elements—central. (c) Single element—offset. (d) Maximum G/T, 5 elements—offset. (e) Normalized conjugate match, 21 elements—offset. (f) Maximum G/T, 21 elements—offset.

TABLE II

FPA NOISE BUDGET

\begin{tabular}{lcc}
\hline Parameter & Value & $\begin{array}{c}\text { Contrib. To } \\
T_{\mathrm{rx}}(\mathrm{K})\end{array}$ \\
\hline Noise from surrounding elements $^{a}$ & $11 \mathrm{~K}$ & 11 \\
Dissipative loss in antenna $^{b}$ & $0.5 \mathrm{~dB}$ & 38 \\
LNA noise figure $^{c}$ & $0.6 \mathrm{~dB}$ & 48 \\
Gain of LNA $^{c}$ & $12.6 \mathrm{~dB}$ & \\
Noise figure of filter and second amp. $^{d}$ & $4.6 \mathrm{~dB}$ & 34 \\
Gain of filter and second amp. $^{d}$ & $17.0 \mathrm{~dB}$ & \\
Focus to receiver cable loss $^{e}$ & $10.0 \mathrm{~dB}$ & 3 \\
Receiver Noise Figure $^{d}$ & $2.0 \mathrm{~dB}$ & 2 \\
\hline Total & & 137 \\
\hline
\end{tabular}

${ }^{a}$ This is the energy picked up by each element that has been radiated from the termination presented by surrounding LNAs. It was calculated from the scattering matrix measured from the array (without the electronics) and an estimated equivalent noise temperature presented by the LNA inputs of $100 \mathrm{~K}$ [50].

${ }^{b}$ Estimated by the FPA supplier [50].

${ }^{c}$ Data sheet for the LNA [54].

${ }^{d}$ Calculated by the designer [55].

${ }^{e}$ Measured prior to installation.

case. The difference between the normalized conjugate match and maximum G/T for 21-elements is $10 \pm 1 \mathrm{~K}$. The $Y_{\text {src }}, Y_{\text {abs }}$ and $T_{\mathrm{rx}}$ values can be combined to find the antenna aperture efficiency using the expression

$$
\eta_{\mathrm{ap}}=\frac{\left(T_{\mathrm{abs}}+T_{\mathrm{rx}}\right)}{Y_{\mathrm{abs}}} \frac{2 k_{\mathrm{B}}\left(Y_{\mathrm{src}}-1\right)}{A \mathrm{~S}} .
$$

The values for the offset element selection weightings range from $41-57 \%$ (Table I). The combined uncertainty in the efficiency is $\pm 10 \%$ in absolute terms and is dominated by the uncertainty in $Y_{\text {src }}$. It is interesting to note that this calculation is relatively insensitive to the coarse estimate of the receiver noise with it contributing only $2-4 \%$ to the total. Higher efficiencies are expected with an FPA where more elements are used.

\section{Comparison of Weighting Cases}

In the measured results there is close correspondence between the normalized conjugate match and the maximum G/T weighting cases in the radiation patterns, the aperture distributions and the G/T measures. The normalized conjugate match has a slightly higher directivity (aperture efficiency) and the maximum $\mathrm{G} / \mathrm{T}$ has slightly better noise performance. As the normalized conjugate match does not take spillover noise into account (assuming identical spillover for the individual elements) the similarity suggests aperture efficiency improvement and not noise reduction dominated both cases.

The maximum $\mathrm{G} / \mathrm{T}$ weighting reduces to the normalized conjugate match when the noise covariance matrix $\mathbf{C}_{T_{\mathrm{sys}}}$ is diagonal. On this instrument the receiver noise temperature is relatively high, hence the dominant diagonal terms in $\mathbf{C}_{T_{\mathrm{sys}}}$. Where the receiver temperature is very low, reduction of spillover is expected to be more dominant for the maximum G/T case and the two weightings would be less similar.

The five-element case showed about half the G/T improvement of the 21-element case (Table I). The difference between the G/T comparison method (Section IV-B) and the on/off M87 result is accounted for by the uncertainty estimates: $2.1 \pm 0.1$ 
and $2.4 \pm 0.7 \mathrm{~dB}$ respectively for maximum $\mathrm{G} / \mathrm{T}$ over single element.

The normalized conjugate match and the maximum gain weightings are shown to be equivalent in certain cases as shown in Appendix B. They have the same relative phase and are equivalent if across the beamformed elements, the radiation efficiencies are equal and the noise temperatures are equal. The dominantly weighted elements are away from the edge of the array and so will have similar radiation efficiencies. Measurements on the FPA show a $T_{\text {sys }}$ variation of up to $2.5 \mathrm{~dB}$. Taking the worst case of this disparity being on two dominant elements, the reduction in the gain is $0.09 \mathrm{~dB}$. That is gain from the normalized conjugate match is within a tenth of a decibel of the maximum gain achievable.

\section{CONCLUSION}

The practical implementation of beamforming an FPA in a prototype radiotelescope has been presented. A normalized conjugate match and the maximum G/T weightings have been applied and evaluated. The results were compared to a single element from the array and a similar moderate improvement in $\mathrm{G} / \mathrm{T}$ was seen in both cases. With lower noise FPAs, a greater difference between the two weightings is expected.

The formulation for the beamforming was presented using a black box approach based on standard beamforming methods. Scattering matrix or similar models are essential for good FPA system design and can provide good predictions of performance [15]. The black box approach shown here demonstrates what can be readily determined from measurements on an installed system alone. It accommodates factors such as any omissions in modelling, such as supporting structures, and drift in the electronic gain amplitude and phases with temperature and over time as well as component failures. The relationship between the black box formulation and a scattering matrix method was also detailed. The black box approach does not separate out the performance of the different components and so is less useful for synthesis or analyzing where the system could be improved.

Beamforming schemes used in an operating radiotelescope are likely to be close to the maximum G/T methods explored here (and in other recent FPA prototypes) because of the importance of sensitivity to the field of radioastronomy. The maximum G/T does not however address other considerations such as sidelobe level, main lobe shape, pattern stability and polarization purity. These may need to be taken into account as well (as in [29], [34]) and would require high dynamic range measurements or accurate modelling of the element radiation patterns. The normalized conjugate match was shown to be useful in the system development due to its simplicity but is unlikely to be appropriate in an operational setting because it does not minimize correlated noise.

The evaluation techniques allowed the antenna system to be characterized with some limitations. The radiation patterns gave an indication of the aperture distribution but more extensive pattern cuts or a holography grid would provide superior resolution and greater confidence in the interpretation of the results. A relatively sensitive technique for comparing $\mathrm{G} / \mathrm{T}$ values as well as a less sensitive method for determining the absolute value of $\mathrm{G} / \mathrm{T}$ were demonstrated. The uncertainty of the latter was

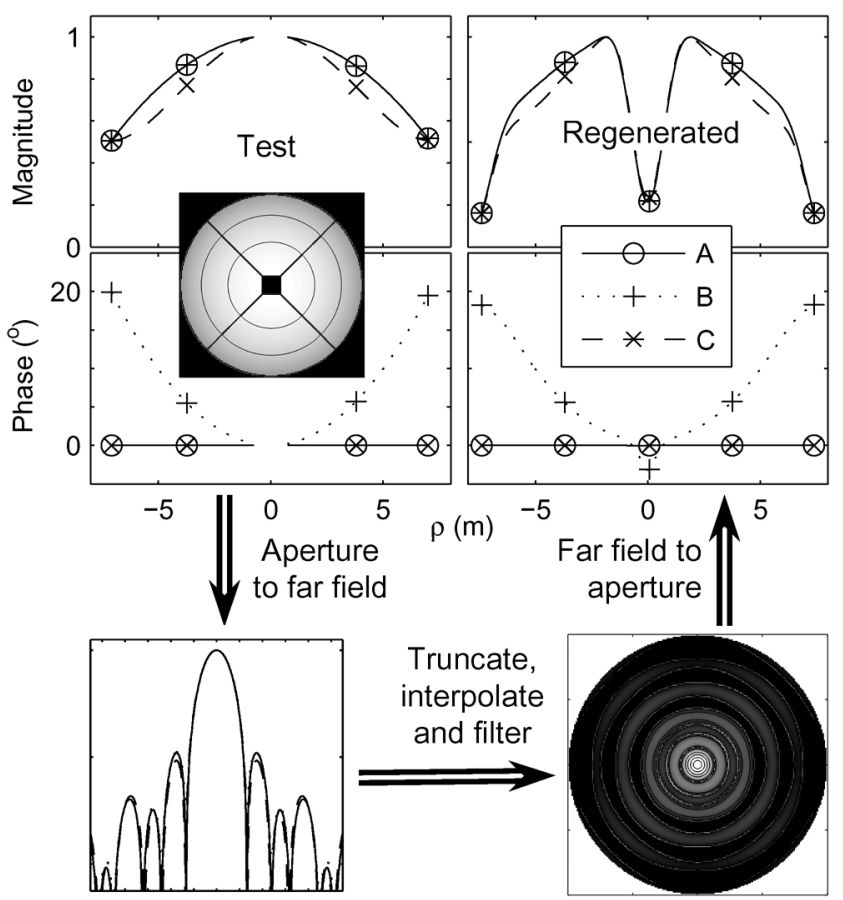

Fig. 8. E-plane cuts of test and regenerated apertures. The $2 \mathrm{D}$ insert shows the FPA and strut blockage used in the model. The parameters for the apertures are: A: $n=1$, flat phase; B: $n=1$ quadratic phase; C: $n=2$, flat phase, all with $6 \mathrm{~dB}$ edge taper.

high due to the limited strength of the source, M87. This uncertainty would be much lower with a stronger source such as the northern hemisphere's Cassiopeia-A. While the noise performance of this system was modest, the beamforming and evaluation techniques are applicable to future systems.

Modelling and design techniques for FPAs are rapidly improving with the efforts from a number of groups. Within CSIRO, FPA development is continuing using another test-bed at the Parkes observatory (where the RFI is much lower) [19], [36], [46].

\section{APPENDIX A \\ APERTURE DISTRIBUTION CALCULATION}

The available data consists of four patterns cuts, equally spaced in the azimuthal coordinate, $\phi$, over eight or ten degree spans in the elevation coordinate, $\theta$, where the coordinate system is aligned with the beam peak. This angle is interpolated as described in Section IV-B and $\phi$, is interpolated using a cubic spline. The aperture distributions are then calculated by applying 2D Fourier transforms to this far-field data. A Hamming window is used to reduce ripples in the aperture distributions.

The validity and limitations of this technique was tested using models of aperture distributions. These include feed and strut blockage. A parabolic taper on a pedestal was used for the test distribution with differing profiles and $6 \mathrm{~dB}$ edge illumination $(C=0.5): E(\rho)=C+(1-C)\left[1-(\rho / a)^{2}\right]^{n}$. Quadratic phase distortion of similar magnitude to the single element results, was introduced in the form $\arg (E(\rho))=20^{\circ}(\rho / a)^{2}$. $a$ is the radius of the dish. Magnitude and phase cuts of the fields in three such test apertures are in Fig. 8 along with the regenerated apertures. The far-field patterns were generated from the 


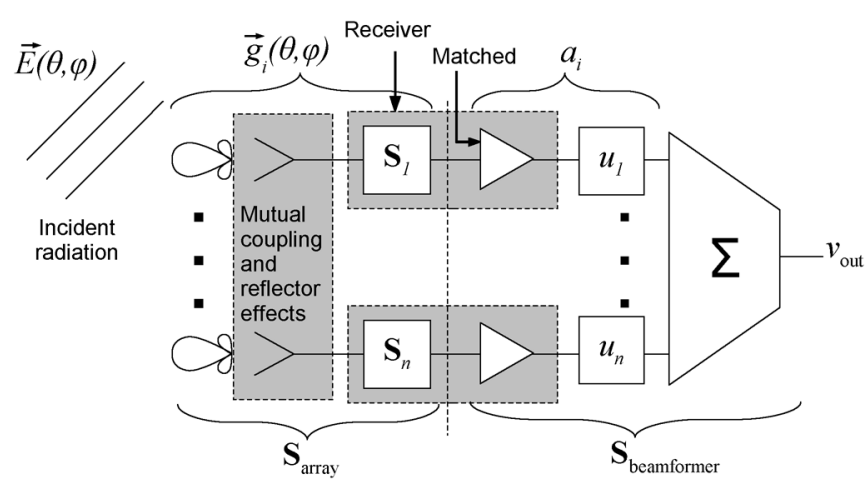

Fig. 9. Scattering matrix model of the array showing the location of the reference plane separating the array to the left and the beamformer to the right. Note the receiver match is on the array side.

test apertures (using the formulation in [56], p283). Comparison of the initial and regenerated apertures show the effect of spatial filtering and little can be determined by the regions around the blocked center of the dish or the edges. Within the annulus of $\rho=2$ to $6 \mathrm{~m}$ however, the test apertures exhibited an RMS phase error of $0.24^{\circ}$ for the quadratic phase case and much less for the flat phase case and 2\% and 5\% RMS amplitude error for the $n=1$ and $n=2$ cases respectively.

\section{APPENDIX B}

\section{NORMALIZED CONJUGATE MATCH AND MAXIMUM GAIN}

Our purpose here is to relate the black-box implementation (Section II) to a conventional scattering matrix representation, which is often used in synthesis. The correspondence between the models is used to demonstrate the conditions under which the normalized conjugate match weighting (black box) is equivalent to the maximum gain weighting (scattering matrix). Particularly noted is the difference in the choice of reference plane dividing the array and the beamformer.

Scattering matrix analyses of arrays is well covered in the literature in a range of different forms (e.g. [57] and [58]). The scattering matrix model of the array is shown in Fig. 9. The receiver electronics (with the LNA having the major effect) is included in the model by splitting each receiver into a passive-reciprocal two-port, $\mathbf{S}_{i}$, representing the network element presented to the array port and an isolating-matched amplifier representing the electronic gain (Fig. 9). The gain is included in the weighting terms $a_{i}$. The $g_{p, i}(\theta, \phi)$ are embedded-element field-patterns for the $\hat{p}$ polarization and the antenna gain (including the receiver match) at the $i^{t h}$ port is $G_{p, i}=4 \pi g_{p, i}^{*} g_{p, i}^{*}$. The voltage signal response to a $\hat{p}$ polarized plane-wave $E_{p}$ in terms of the scattering matrix model is (cf. (1))

$$
v_{\text {out }}(t)=\frac{\lambda}{\sqrt{2 \eta_{0}}} E_{p}(t) \mathbf{g}_{p}^{T} \mathbf{a}
$$

where $\mathbf{a}$ and $\mathbf{g}$ are the weight and embedded-element field-pattern column vectors.
The choice of reference plane results in the field patterns from the black box and scattering matrix models to be related by complex terms that are constant over the sphere. Equivalent weightings from the two models (i.e. producing the same output for the same incident radiation) obey the identity $\mathbf{e}_{p}^{T} \mathbf{w}=\mathbf{g}_{p}^{T} \mathbf{a}$.

A consequence of the field patterns from the two models being proportional to each other is their directivities are the same

$$
\begin{aligned}
D_{p, i} & =4 \pi \frac{e_{p, i}^{*} e_{p, i}}{\iint_{\Omega} e_{p, i}^{*} e_{p, i}+e_{q, i}^{*} e_{q, i} d \Omega} \\
& =4 \pi \frac{g_{p, i}^{*} g_{p, i}}{\iint_{\Omega} g_{p, i}^{*} g_{p, i}+g_{q, i}^{*} g_{q, i} d \Omega} .
\end{aligned}
$$

The denominator of (20) is unity from the definition of the $e_{p, i}$ ((2)). Consider (21) in terms of the scattering matrix model in transmit. The denominator is the ratio of total power radiated and power available for the $i^{\text {th }}$ port only excited. By comparing (20) and (21), the relationship between the $\mathbf{g}_{p}$ and the $\mathbf{e}_{p}$ is

$$
g_{p, i}(\theta, \phi)=\sqrt{\eta_{\text {radiated }, i}} \exp \left(\mathrm{j} \Psi_{i}\right) e_{p, i}(\theta, \phi)
$$

and between $\mathbf{w}$ and $\mathbf{a}$

$$
w_{p, i}=\sqrt{\eta_{\text {radiated }, i}} \exp \left(\mathrm{j} \Psi_{i}\right) a_{p, i} .
$$

where $\Psi_{i}$ is an (undetermined) phase.

The maximum gain condition, that is the maximum power delivered to the beamformer output, given the constraint that $\mathbf{a}^{H} \mathbf{a}$ is constant, is given by the conjugate match to the signals from the array port, $\mathbf{a}=\mathbf{g} \alpha$. Inserting this into (23), $w_{p, i}=$ $\eta_{\text {radiated }, i} e_{p, i}^{*}$.

If both $\eta_{\text {radiated, } i}$ and $\left[\mathbf{C}_{T_{\mathrm{sys}}}\right]_{i i}$ are constant across the array, the normalized-conjugate-match condition is equivalent to the maximum gain condition.

\section{ACKNOWLEDGMENT}

This work was facilitated by the ASKAP project and its predecessors and has only been possible through the contributions of the large team that contributed to the prototype radiotelescope [22]. ASTRON contributed both with the FPA itself and with advice during this project. In particular M. Ivashina and B. Woestenburg have provided data and advice on evaluating the FPA. The authors thank the anonymous reviewers for their comments resulting in an improved paper. D. B. Hayman thanks S. Hay, J. O'Sullivan, and M. Kesteven for their additional encouragement and advice during this project.

\section{REFERENCES}

[1] J. R. Fisher and R. F. Bradley, "Full sampling array feeds for radio telescopes," in Proc. SPIE, Radio Telescopes, H. R. Butcher, Ed., Jul. 2000, vol. 4015, pp. 308-318, SPIE.

[2] M. V. Ivashina and J. D. B. A. van Ardenne, "A way to improve the field of view of the radiotelescope with a dense focal plane array," in Proc. 12th Int. Conf. Microwave and Telecommunication Technology, 2002, pp. 278-281. 
[3] Multi-Feed Systems for Radio Telescopes, ser. Astron. Soc. Pac. Conf., D. T. Emerson and J. M. Payne, Eds. Tucson, AZ: , May 16-18, 1994, vol. 75.

[4] L. Staveley-Smith, W. E. Wilson, T. S. Bird, M. J. Disney, R. D. Ekers, K. C. Freeman, R. F. Haynes, M. W. Sinclair, R. A. Vaile, R. L. Webster, and A. E. Wright, "The Parkes 21 CM multibeam receiver," Publ. Astron. Soc. Aust., vol. 13, pp. 243-248, Nov. 1996.

[5] J. F. Johansson, "Fundamental limits for focal-plane array efficiency," in Multi-Feed Systems for Radio Telescopes, ser. Astron. Soc. Pac. Conf.. Tucson, AZ: , May 16-18, 1994, vol. 75, pp. 34-41 [Online]. Available: http://adsabs.harvard.edu/abs/1995ASPC ...75 ...34J

[6] B. Veidt, Focal-Plane Array Architectures: Horn Clusters vs. PhaseArray Techniques International Square Kilometre Array Steering Committee, SKA Memo 71, 2006 [Online]. Available: http://www.skatelescope.org/PDF/memos/71_Veidt.pdf

[7] P. Loux and R. Martin, "Efficient aberration correction with a transverse focal plane array technique," in Proc. IRE Int. Convention Record, 1964, vol. 12, pp. 125-131, IEEE.

[8] R. Padman, "Optical fundamentals for array feeds," Multifeed Systems for Radio Telescopes, ser. Astron. Soc. Pac. Conf., vol. 75, 1995 [Online]. Available: http://adsabs.harvard.edu/abs/1995ASPC...75 ....3P

[9] K. F. Warnick, B. D. Jeffs, J. Landon, J. Waldron, D. Jones, A. Stemmons, J. R. Fisher, R. Norrod, and R. Bradley, "BYU/NRAO 19 element L-band focal plane array feed-Sensitivity, efficiency, and RFI mitigation," in Eur. Conf. on Antennas and Propagation (EuCAP), Edinburgh, UK, Nov. 2007 [Online]. Available: http://wiki.gb.nrao.edu/ pub/Electronics/ResultPresentations/ArrayFeed E uCAP_Nov07.pdf

[10] K. F. Warnick, B. D. Jeffs, J. Landon, J. Waldron, D. Jones, J. R. Fisher, and R. Norrod, "Beamforming and imaging with the BYU/ NRAO 1-band 19-element phased array feed," in Proc. ANTEM Symp., Banff, AB, Feb. 15-18, 2009, pp. 1-4.

[11] C. Craeye, A. B. Smolders, A. G. Tijhuis, and D. H. Schaubert, "Computation of finite array effects in the framework of the square kilometer array project," in Proc. Inst. Elect. Eng. Int. Conf. on Antennas and Propagation (ICAP), Manchester, Apr. 17-20, 2001, vol. 1, pp. 298-301.

[12] J. Simons, J. G. B. de Vaate, M. V. Ivashina, M. Zuliani, V. Natale, and N. Roddis, "Design of a focal plane array system at cryogenic temperatures," in Proc. Eur. Conf. on Antennas and Propagation (EuCAP), Nice, Nov. 6-10, 2006, pp. 1-6.

[13] M. A. W. Verheijen, T. A. Oosterloo, W. A. van Cappellen, L. Bakker, M. V. Ivashina, and J. M. van der Hulst, "Apertif, a focal plane array for the WSRT," in The Evolution of Galaxies Through the Neutral Hydrogen Window, Aug. 2008, vol. 1035, pp. 265-271, Am. Inst. Phys. Conf. Proc. series.

[14] M. V. Ivashina, M. N. M. Kehn, P. S. Kildal, and R. Maaskant, "Decoupling efficiency of a wideband Vivaldi focal plane array feeding a reflector antenna," IEEE Trans. Antennas Propag., vol. 57, no. 2, pp. 373-382, Feb. 2009.

[15] M. V. Ivashina, O. A. Iupikov, R. Maaskant, W. A. van Cappellen, L. Bakker, and T. Oosterloo, "Off-axis beam performance of focal plane arrays for the westerbork synthesis radio telescope-Initial results of a prototype system," in IEEE AP-S Int. Symp. Digest, Charleston, SC, Jun. 1-5, 2009, pp. 1-4.

[16] B. Veidt and P. Dewdney, "Development of a phased-array feed demonstrator for radio telescopes," presented at the ANTEM Symp., SaintMalo, France, Jun. 2005.

[17] B. Veidt, T. Burgess, R. Messing, G. Hovey, and R. Smegal, "The DRAO phased array feed demonstrator: Recent results," in Proc. ANTEM Symp., Banff, AB, Feb. 15-18, 2009, pp. 1-4.

[18] P. E. Dewdney, P. J. Hall, R. T. Schilizzi, and T. J. L. W. Lazio, "The square kilometre array," Proc. IEEE, vol. 97, no. 8, pp. 1482-1496, Aug. 2009.

[19] D. R. DeBoer, R. G. Gough, J. D. Bunton, T. J. Cornwell, R. J. Beresford, S. Johnston, I. J. Feain, A. E. Schinckel, C. A. Jackson, M. J. Kesteven, A. Chippendale, G. A. Hampson, J. D. O'Sullivan, S. G. Hay, C. E. Jacka, T. W. Sweetnam, M. C. Storey, L. Ball, and B. J. Boyle, "Australian SKA pathfinder: A high-dynamic range wide-field of view survey telescope," Proc. IEEE, vol. 97, no. 8, pp. 1507-1521, Aug. 2009.

[20] D. B. Hayman and T. Cornwell, "NTD THEA tile measurements," presented at the 3rd Int. Focal Plane Array Workshop, Mar. 2007.

[21] J. O'Sullivan, R. Gough, D. B. Hayman, A. Grancea, C. Granet, S. Hay, and J. Kot, "Recent focal plane array developments for the Australian SKA Pathfinder," presented at the Int. Symp. on Microwave and Optical Technology (ISMOT), Dec. 2007.
[22] D. Hayman, R. Beresford, J. Bunton, C. Cantrall, T. Cornwell, A. Grancea, C. Granet, J. Joseph, M. Kesteven, J. O'Sullivan, J. Pathikulangara, T. Sweetnam, and M. Voronkov, "The NTD interferometer: A phased array feed test bed," presented at the URSI Workshop on Applications of Radio Science, 2008 [Online]. Available: http://www.ncrs.org.au/wars/wars2008/Hayman

[23] D. B. Hayman, T. S. Bird, P. Hall, and K. Esselle, "Evaluation of beamforming radioastronomy focal plane arrays," presented at the UNSC/ URSI, 2009.

[24] Y. T. Lo, S. W. Lee, and Q. H. Lee, "Optimization of directivity and signal-to-noise ratio of an arbitrary antenna array," Proc. IEEE, vol. 54, no. 8, pp. 1033-1045, 1966.

[25] S. P. Applebaum, "Adaptive arrays," IEEE Trans. Antennas Propag., vol. 24, no. 5, pp. 585-598, Sep. 1976.

[26] B. Minnett and H. Thomas, "A method of synthesizing radiation patterns with axial symmetry," IEEE Trans. Antennas Propag., vol. 14, no. 5, pp. 654-656, Sep. 1966.

[27] M. V. Ivashina, J.-G. bij de Vaate, R. Braun, and J. D. Bregman, "Focal plane arrays for large reflector antennas: First results of a demonstrator project," presented at the SPIE Astronomical Telescopes and Instrumentation, Glasgow, Scotland, U.K., 2004.

[28] M. V. Ivashina, J. Simons, and J. G. B. Vaate, "Efficiency analysis of focal plane arrays in deep dishes," Exp. Astron., vol. 17, no. 1-3, pp. 149-162, Jun. 2004.

[29] T. S. Bird, "Contoured-beam synthesis for array-fed reflector antennas by field correlation," Proc. Inst. Elect. Eng. Microw. Opt. Antennas, vol. 129, pp. 293-298, Dec. 1982.

[30] P. Lam, S.-W. Lee, D. Chang, and K. Lang, "Directivity optimization of a reflector antenna with cluster feeds: A closed-form solution," IEEE Trans. Antennas Propag., vol. 33, no. 11, pp. 1163-1174, 1985.

[31] T. S. Bird and D. B. Hayman, "Focal-plane array concepts for the Parkes radio telescope," presented at the URSI General Assembly, Lille, France, Sep. 1996.

[32] W. Brisken and C. Craeye, Focal Plane Array Beam-Forming and Spill-Over Cancellation Using Vivaldi Antennas National Radio Astronomy Observatory, EVLA Memo 69, Jan. 2004 [Online]. Available: http://www.aoc.nrao.edu/evla/geninfo/memoseries/evlamemo69.pdf

[33] C. K. Hansen, K. F. Warnick, B. D. Jeffs, J. R. Fisher, and R. Bradley, "Interference mitigation using a focal plane array," Radio Sci., vol. 40, p. RS5S16, Jun. 2005.

[34] B. D. Jeffs, K. F. Warnick, J. Landon, J. Waldron, D. Jones, J. R. Fisher, and R. D. Norrod, "Signal processing for phased array feeds in radio astronomical telescopes," IEEE J. Sel. Topics Signal Process., vol. 2, no. 5, pp. 635-646, Oct. 2008.

[35] K. F. Warnick and M. A. Jensen, "Optimal noise matching for mutually coupled arrays," IEEE Trans. Antennas Propag., vol. 55, no. 6, pp. 1726-1731, Jun. 2007.

[36] S. G. Hay, J. D. O'Sullivan, J. S. Kot, C. Granet, A. Grancea, A. R. Forsyth, and D. B. Hayman, "Focal plane array development for ASKAP (Australian SKA Pathfinder)," in Proc. Eur. Conf. on Antennas and Propagation (EuCAP), Edinburgh, Nov. 2007, pp. $1-5$.

[37] Standard Definitions of Terms for Antennas, IEEE Std. 145-1993, Mar. 1993.

[38] A. Waldman and G. J. Wooley, "Noise temperature of a phased array receiver," Microw. J., vol. 9, pp. 89-96, Sep. 1966.

[39] E. Jacobs, "A figure of merit for signal processing reflector antennas," IEEE Trans. Antennas Propag., vol. 33, no. 1, pp. 100-101, Jan. 1985.

[40] J. J. Lee, "G/T and noise figure of active array antennas," IEEE Trans. Antennas Propag., vol. 41, no. 2, pp. 241-244, Feb. 1993.

[41] K. F. Warnick and B. D. Jeffs, "Gain and aperture efficiency for a reflector antenna with an array feed," IEEE Antennas Wireless Propag. Lett., vol. 5, no. 1, pp. 499-502, Dec. 2006.

[42] S. G. Hay, "FPA modelling concepts at CSIRO," in Focal Plane Array Workshop, Dwingeloo, The Netherlands, Jun. 2005 [Online]. Available: http://www.astron.nl/fpaworkshop2005/, Astron

[43] K. F. Warnick and M. A. Jensen, "Effects of mutual coupling on interference mitigation with a focal plane array," IEEE Trans. Antennas Propag., vol. 53, no. 8, pp. 2490-2498, Aug. 2005.

[44] D. K. Cheng and F. I. Tseng, "Gain optimization for arbitrary antenna arrays," IEEE Trans. Antennas Propag., vol. AP-13, pp. 973-974, Nov. 1965.

[45] S. J. Wijnholds, A. G. Bruyn, J. D. Bregman, and J. G. B. Vaate, "Hemispheric imaging of galactic neutral Hydrogen with a phased array antenna system," Exp. Astron., vol. 17, no. 1-3, pp. 59-64, Jun. 2004. 
[46] J. D. O'Sullivan, F. Cooray, C. Granet, R. Gough, S. Hay, D. B. Hayman, M. Kesteven, J. Kot, A. Grancea, and R. Shaw, "Phased array feed development for the Australian SKA Pathfinder," presented at the URSI General Assembly, Sep. 2008.

[47] R. C. Hansen, Phased Array Antennas, K. Chang, Ed. New York: Wiley, 1998.

[48] O. M. Bucci, C. Gennarelli, and C. Savarese, "Optimal interpolation of radiated fields over a sphere," IEEE Trans. Antennas Propag., vol. 39, no. 11, pp. 1633-1643, Nov. 1991.

[49] M. Ott, A. Witzel, A. Quirrenbach, T. P. Krichbaum, K. J. Standke, C. J. Schalinski, and C. A. Hummel, "An updated list of radio flux density calibrators," Astron. Astrophys., vol. 284, pp. 331-339, Apr. 1994.

[50] E. E. M. Woestenburg, Personal Communication. 2007.

[51] A. W. Rudge and M. J. Withers, "New technique for beam steering with fixed parabolic reflectors (Wide angle microwave antenna radiation beam steering with fixed parabolic reflectors, using adaptive primary feed for intercepted field spatial Fourier transformation)," Proc. Inst. Elect. Eng., vol. 118, pp. 857-863, Jul. 1971.

[52] P.-S. Kildal, Foundations of Antennas: A Unified Approach. Sweden: Studentlitteratur, 2000.

[53] E. E. M. Woestenburg and K. F. Dijkstra, "Noise characterization of a phased array tile," in Proc. Eur. Microwave Conf. (EuMC), 2003, vol. 1 , pp. 363-366.

[54] "CGY2106TS Dual LNA Data Sheet," Philips Semiconductors, 2000.

[55] A. Grancea, Personal Communication. 2006.

[56] W. L. Stutzman and G. A. Thiel, Antenna Theory and Design, 2nd ed. New York: Wiley, 1998.

[57] S. Stein, "On cross coupling in multiple-beam antennas," IEEE Trans. Antennas Propag., vol. AP-10, no. 5, pp. 548-557, Sep. 1962.

[58] J. W. Wallace and M. A. Jensen, "Mutual coupling in MIMO wireless systems: A rigorous network theory analysis," IEEE Trans Wireless Commun., vol. 3, no. 4, pp. 1317-1325, 2004.

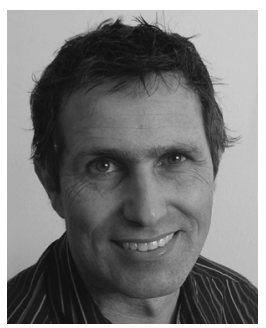

Douglas Brian Hayman (M'93) was born in Misawa, Japan, in 1964. He received the B.Sc. degree in pure mathematics and physics in 1986 and the B.E. degree (electrical, Hons.) in 1988, both from the University of Sydney, Sydney, Australia.

In 1988, he joined Radio Transmission Engineering, working on FM broadcast transmitters and microwave links. From 1990 to 1992, he was with the satellite company, AUSSAT, working in earth station engineering. In December in 1992, he joined the Commonwealth Scientific and Industrial Research Organisation (CSIRO), in Sydney, Australia, where is he currently a Senior Research Engineer. He has focused on antenna metrology and led a comprehensive upgrade of CSIRO's antenna measurement facility hardware and software. He has also designed components such as waveguide rotary joints and orthomode transducers and managed a number of antenna related projects. Most recently he has been involved in the ASKAP focal plane array work and, since 2003, he has been undertaking a part time Ph.D. in the field of focal plane array beamforming and evaluation at Macquarie University, NSW, Australia.

Mr. Hayman has served as a reviewer for the IEEE TRANSACTIONS ON ANTENNAS AND PROPAGation since 2005 and for the Australian Symposium on Antennas since 2006.

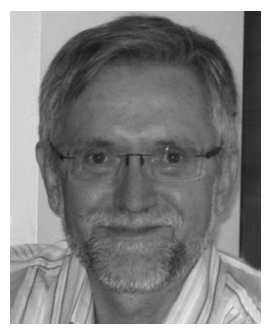

Trevor S. Bird (S'71-M'76-SM'85-F'97) received the B.App.Sc., M.App.Sc., and Ph.D. degrees from the University of Melbourne, Melbourne, Australia, in 1971,1973 , and 1977 , respectively.

From 1976 to 1978 , he was a Postdoctoral Research Fellow at Queen Mary College, University of London, London, U.K., followed by five years as a Lecturer in the Department of Electrical Engineering at James Cook University of North Queensland. During 1982 and 1983, he was a consultant at Plessey Radar, U.K., and in December 1983 he joined the Commonwealth Scientific and Industrial Research Organisation (CSIRO) in Sydney, Australia. He has held several positions with CSIRO and is currently a CSIRO Fellow and Chief Scientist in the CSIRO Information and Communication Technologies Centre. He is also an Adjunct Professor at Macquarie University, Sydney.

Dr. Bird is a Fellow of the Australian Academy of Technological and Engineering Sciences, the IEEE, the Institution of Engineering and Technology
(IET), London, U.K., and is an Honorary Fellow of the Institution of Engineers, Australia. He has published widely in the areas of electromagnetics and antennas, particularly related to waveguides, horns, reflectors, wireless and satellite communication applications, and he holds twelve patents. In 1988, 1992, 1995, and 1996 he received the John Madsen Medal of the Institution of Engineers, Australia for the best paper published annually in the Journal of Electrical and Electronic Engineering, Australia, and in 2001 he was co-recipient of the H. A. Wheeler Applications Prize Paper Award of the IEEE Antennas and Propagation and Society. He was awarded a CSIRO Medal in 1990 for the development of an Optus-B satellite spot beam antenna and again in 1998 for the multibeam antenna feed system for the Parkes radio telescope. He received an IEEE Third Millennium Medal in 2000 for outstanding contributions to the IEEE New South Wales Section. Engineering projects that he played a major role in were given awards by the Society of Satellite Professionals International (New York) in 2004, the Engineers Australia in 2001, and the Communications Research Laboratory, Japan, in 2000. In 2003 he was awarded a Centenary Medal for service to Australian society in telecommunications and also named Professional Engineer of the Year by the Sydney Division of Engineers Australia. His biography is listed in Who's Who in Australia. He was a Distinguished Lecturer for the IEEE Antennas and Propagation Society from 1997 to 1999. Chair of the New South Wales joint AP/MTT Chapter from 1995 to 1998, and again in 2003, Chairman of the 2000 Asia Pacific Microwave Conference, Member of the New South Wales Section Committee from 1995-2005 and was Vice-Chair and Chair of the Section in 1999-2000 and 2001-2002 respectively, Associate Editor of the IEEE TRANSACTIONS ON ANTENNAS AND PROPAGATION from 2001 to 2004, a member of the Administrative Committee of the IEEE Antennas and Propagation Society from 2003-2005, and a member of the College of Experts of the Australian Research Council (ARC) from 2006-2007. He has been a member of the technical committee of numerous conferences including JINA, ICAP, AP2000, IRMMW-THz and the URSI Electromagnetic Theory Symposium. Currently, he is member of the Editorial Boards of the IEEE TRANSACTIONS ON MiCROWAVE THEORY AND TECHNIQUES and the Journal of Infrared, Millimeter and Terahertz Waves. He has been Editor-in-Chief of the IEEE TRANSACTIONS ON ANTENNAS AND PROPAGATION since 2004.

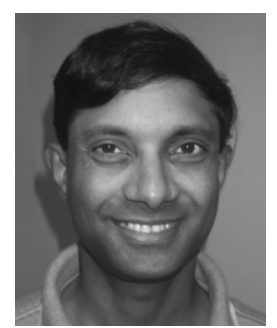

Karu P. Esselle (M'92-SM'96) received the B.Sc. degree in electronic and telecommunication engineering (First Class Honors) from the University of Moratuwa, Sri Lanka, in 1983 and the M.A.Sc. and Ph.D. degrees in electrical engineering from the University of Ottawa, Ottawa, ON, Canada, in 1987 and 1990, respectively.

$\mathrm{He}$ is a Professor in electronic engineering, Macquarie University, Sydney. He was the Immediate Past Associate Dean - Higher Degree Research and the Founding Director of Postgraduate Research Committee in the Division of Information and Communication Sciences. He held these positions 2003 - 2008 and was also a member of the Division Executive. He served in all Macquarie University HDR-related committees at the highest level. He is the Director of Electromagnetic and Antenna Engineering, and the Deputy Director of the Research Centre on Microwave and Wireless Applications (CMWA), which was recently expanded after recognised as a Concentration of Research Excellence. He has been invited to serve as an international expert/research grant assessor by several overseas nationwide research funding bodies form the Netherlands, Finland, Hong-Kong and Chile. In Australia, he has been invited to assess grant applications submitted to the nation's most prestigious schemes such as Australian Federation Fellowships and Australian Laureate Fellowships. His industry experience includes full-time employment as Design Expert by the Hewlett Packard Laboratory, USA, and several consultancies for local and international companies, including Cisco Systems (USA), Optus Networks, Locata (USA)/QX Corporation, ResMed, FundEd and Katherine-Werke (Germany) through Peter-Maxwell Solicitors. $\mathrm{He}$ was an Assistant Lecturer at the University of Moratuwa, a Canadian Government laboratory Visiting Postdoctoral Fellow at Health Canada, a Visiting Professor of the University of Victoria and a Visiting Scientist of the Information and Communication Technologies Centre (ICT Centre), Commonwealth Scientific and Industrial Research Organisation (CSIRO), Sydney, Australia. He is an Editor of the International Journal of Antennas and Propagation. He has authored about 250 scientific publications, including six invited book chapters and over fifteen invited conference presentations. His current research interests include metamaterials and their microwave applications, photonic crystals and photonic band gap (PBG)/electromagnetic band gap (EBG) structures, millimeter-wave EBG MMIC devices, antennas based on EBG, periodic structures including frequency selective surfaces, 
antennas for mobile and wireless communication systems including $\mathrm{WiFi}$, WiMAX, HyperLAN, and ultrawideband systems, antennas for multi-signal location and navigation systems, dielectric-resonator (DR) antennas, broadband and multi-band printed antennas, smart antenna systems, hybrid antennas, theoretical methods, lens and focal-plane-array antennas for radio astronomy, moment methods, FDTD methods for periodic structures and closed-form Green's functions for layered structures. His research activities are posted in the web at www.elec.mq.edu.au/celane/.

Prof. Esselle's recent awards include the 2009 Vice Chancellor's Award for Excellence in Higher Degree Research Supervision (the first such award ever offered in Macquarie University) and 2004 (Inaugural) Innovation Award for best invention disclose. Since 2002, he was involved with research grants and contracts worth about five million dollars, and his research team members attracted further grants worth about a million dollars. The CELANE, which he founded, has provided a stimulating research environment for a strong team of researchers including six postdoctoral fellows. His mentees have been awarded 6 extremely competitive postdoctoral fellowships. Nine international experts who examined the theses of his recent five Ph.D. graduates ranked them in the top 5\% or $10 \%$ in the world. He has served on Technical Program Committees or International Committees for many International Conferences. He will be chairing the Technical Program Committee of APMC 2011; he was the Publicity Chair of the APMC 2000. He is the Chair of the IEEE New South Wales (NSW) MTT/AP Joint Chapter, Editor of MQEC, the past Chair of the Educational Committee of the IEEE NSW, and a member of the IEEE NSW Committee.

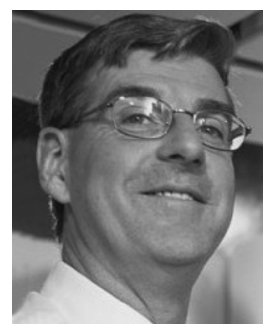

Peter J. Hall was born in Hobart, Tasmania, in 1957 He was received the B.Eng. degree from the Tasmanian College of Advanced Education, in 1980, and the B.Sc. (Hons.) and Ph.D. degrees from the University of Tasmania, in 1981 and 1985 , respectively. His postgraduate study was in the field of radio astronomy, particularly the development of high time resolution polarimeter spectrometers.

He began his professional career in 1985 with a Postdoctoral Fellowship at the Commonwealth Scientific and Industrial Research Organization
(CSIRO), Parkes radio telescope, Sydney, Australia, implementing a novel system for observing the encounter of the Giotto spacecraft with Halley's Comet. He moved to the University of Sydney School of Electrical Engineering as a Lecturer in 1987, prior to re-joining CSIRO in 1989, this time as a Research Scientist and Group Leader responsible for on-site commissioning of the new Australia Telescope Compact Array (ATCA) at Narrabri, in northwestern New South Wales. He performed a similar role during a major upgrade of the Parkes radio telescope in the mid-1990s, the upgrade allowing the telescope to support the Galileo mission to Jupiter while observing the southern sky with a new 13-beam receiver. With a long-time interest in millimeter-wave astronomy, he was a prime instigator of, and system scientist during, a major extension of the ATCA to $100 \mathrm{GHz}$ operation. In 1999 he became Program Leader for CSIRO's Square Kilometre Array (SKA) endeavour, being responsible for developing the scientific and engineering concepts surrounding wide-field astronomy, as well as the initial Australian submission to host the SKA. With a growing role in the global SKA project, he accepted an invitation in 2003 to become the first International Project Engineer, a position he held until 2008. During this time he played a leading role in the establishment of a Reference Design for the SKA, the setting of initial specifications for the instrument, and the formulation of an international design phase now underway. In 2008 he accepted an invitation to join Curtin University of Technology in Perth as its Foundation Professor of Radio Astronomy Engineering. In this capacity he is also Co-Director of the Curtin Institute of Radio Astronomy and a Deputy Director of the International Centre for Radio Astronomy Research (ICRAR).

Professor Hall is a Fellow of the Institution of Engineers (Australia) and a Member of the Institution of Engineering and Technology (IET), London, U.K. 